\title{
CARACTERIZAÇÃO DE USUÁRIOS HIPERTENSOS E ADESÃO AO TRATAMENTO EM UNIDADE DE SAÚDE DA FAMÍLIA
}

Bruno Henrique dos Santos Alves', Monalisa Abila Prado ${ }^{1}$, Nadielly Codonho Góes ${ }^{1}$, Lúcia Marinilza Beccaria², Cláudia Bernardi Cesarino ${ }^{2}$

RESUMO: Este estudo caracterizou o perfil sociodemográfico de 100 usuários hipertensos, entre 500 cadastrados em Unidades de Saúde da Família e identificou fatores associados ao abandono do tratamento. Os dados foram coletados entre julho e outubro de 2011 por meio de visita domiciliar, agrupados em planilha do programa Microsoft Office Excel 2007 e analisados utilizado estatística descritiva e aplicação do teste qui-quadrado. Majoritariamente os hipertensos eram mulheres entre 50 e 69 anos com sobrepeso. Verificou-se que a maioria dos usuários não abandonou o tratamento, mas o realizava em local diverso da unidade. Os motivos relacionados ao abandono ou seguimento inadequado foram a realização do tratamento com cardiologista em outros serviços de saúde, dificuldade de acesso à unidade de saúde e considerar desnecessário o tratamento. Ressalta-se a importância de identificar os motivos para o abandono do tratamento e seu seguimento na unidade de origem para implantação de intervenção. PALAVRAS-CHAVE: Conhecimentos, atitudes e prática em saúde; Tratamento; Hipertensão; Saúde da família.

\section{CHARACTERIZATION OF HYPERTENSIVE SERVICE USERS AND COMPLIANCE WITH TREATMENT IN A FAMILY HEALTH UNIT}

ABSTRACT: This study describes the socio-demographic profile of 100 hypertensive service users, out of 500 registered in Family Health Units. It identified factors associated with abandoning treatment. Data was collected between July and December 2011 through home visiting, was grouped in Microsoft Office Excel spreadsheets, and was analyzed with descriptive statistics and the application of the chi-square test. The majority of the hypertensives were overweight women between 50 and 69 years of age. It was ascertained that the majority of the service users had not abandoned treatment, but were, rather, having it away from the unit. The motives related to abandoning treatment or failing to follow treatment instructions properly were carrying out treatment with a cardiologist in another health service, difficulty of access to the health unit, and considering the treatment un-necessary. The study highlights the importance of identifying the reasons for abandoning treatment or its proper following in the original unit, so as to improve intervention.

KEYWORDS: Knowledge, attitudes and practice in health; Treatment; Hypertension; Family health.

\section{CARACTERIZACIÓN DE USUARIOS HIPERTENSOS Y ADESIÓN AL TRATAMIENTO EN UNIDAD DE SALUD DE LA FAMILIA}

RESUMEN: Este estudio caracterizó el perfil sociodemográfico de 100 usuarios hipertensos, entre 500 cadastrados en Unidades de Salud de la Familia e identificó factores asociados al abandono del tratamiento. Los datos fueron recogidos entre julio y diciembre de 2011 por medio de visita domiciliar, agrupados en planilla del programa Microsoft Office Excel 2007 y analizados utilizádose estadística descriptiva y aplicación del test "qui-quadrado". Los hipertensos eran en la mayoría mujeres entre 50 y 69 años con sobrepeso. Se verificó que la mayor parte de los usuarios no abandonó el tratamiento, pero lo realizava en local diverso de la unidad. Los motivos relacionados al abandono o realización inadecuada fueron la realización del tratamiento con cardiologista en otros servicios de salud, dificultad de acceso a la unidad de salud y considerar desnecesario el tratamiento. Se destaca la importancia de identificar los motivos para el abandono del tratamiento y su realización en la unidad de origen para implantación de intervención.

PALABRAS CLAVE: Conocimientos, actitudes y práctica en salud; Tratamiento; Hipertensión; Salud de la Familia.

${ }^{1}$ Enfermeiro.

${ }^{2}$ Enfermeira. Doutora em Enfermagem. Professora do Departamento de Enfermagem Geral da Faculdade de Medicina de São José do Rio Preto. 


\section{INTRODUÇÃO}

O Programa de Saúde da Família surgiu no Brasil como reorientação do modelo assistencial brasileiro, em conformidade com os princípios do SUS ${ }^{(1-2)}$. A Hipertensão Arterial Sistêmica (HAS) é considerada um dos principais problemas de saúde pública, com desenvolvimento clínico lento e assintomático, com diversos fatores de risco que dificultam o controle e pode levar a várias complicações ${ }^{(3)}$. Este agravo atinge aproximadamente $22 \%$ da população brasileira acima de vinte anos, sendo responsável por $80 \%$ dos casos de acidente cérebro vascular, $60 \%$ dos casos de infarto agudo do miocárdio e $40 \%$ das aposentadorias precoces, além de significar um custo de 475 milhões de reais gastos com 1,1 milhões de internações por ano ${ }^{(4)}$.

Por ser uma doença crônica, não há cura, porém há controle. A grande problemática é o fato dos indivíduos descobrirem a doença quando apresentam complicações graves ${ }^{(5)}$. Sabe-se, também, que a não-adesão ao tratamento da HAS é um dos problemas enfrentados, pois leva ao aumento de morbimortalidade dos indivíduos por não conseguir controlá-la $\mathrm{l}^{(6)}$. Os enfermeiros e membros da equipe de Unidades de Saúde da Família vivenciam que parte dos pacientes hipertensos não apresenta adesão ao tratamento anti-hipertensivo, portanto devem utilizar estratégias para superar este desafio em busca de melhor qualidade de vida aos hipertensos ${ }^{(1)}$.

Frequentemente o enfermeiro identifica os usuários diagnosticados como hipertensos que abandonaram ou não seguem adequadamente o tratamento, levantando os possíveis fatores relacionados, a fim de contribuir com a melhoria da adesão. Dessa forma, o presente estudo objetivou caracterizar o perfil sociodemográfico dos usuários hipertensos cadastrados em Unidades de Saúde da Família e identificar os fatores que levaram ao abandono do tratamento ou não seguimento adequado do tratamento.

\section{MÉTODO}

Pesquisa documental, descritiva e retrospectiva, pesquisa documental em prontuário e visitas domiciliares. Foi realizada em duas Unidades de Saúde da Família do Município de Mirassol, Estado de São Paulo, com população de 53.792 habitantes no ano de 2009 em área de unidade territorial de $243.161 \mathrm{Km}^{(7)}$. As unidades possuem 1.006 usuários hipertensos cadastrados e neste estudo foram identificadas como Unidade A e Unidade B, sendo que a Unidade A possuía 510 hipertensos e a B constam 496.
O estudo foi desenvolvido em duas etapas. Na primeira, realizou-se a caracterização de 500 usuários hipertensos (250 hipertensos cadastrados em cada unidade) quanto ao gênero, idade, indice de massa corporea (IMC), raça, cor, estado civil e escolaridade, isto por meio da análise de prontuário desses usuários. Na segunda etapa, selecionouse destes, de forma aleatória, 50 usuários hipertensos de cada unidade que abandonaram o tratamento da hipertensão ou não seguiam adequadamente o tratamento anti-hipertensivo e foram realizadas visitas domiciliares e entrevistas com os mesmos, com questões relacionadas aos fatores que interferiram no abandono ou seguimento inadequado do tratamento anti-hipertensivo.

A definição de seguimento inadequado do tratamento foi o não comparecimento às consultas na Unidades de Saúde da Família dentro de 12 meses até 23 meses e o abandono do tratamento foi o não comparecimento às consultas em período igual ou maior a 24 meses.

Os dados foram coletados no período de julho a dezembro de 2011 e agrupados na planilha do Programa Microsoft Office Excel 2007. Para análise foi utilizado estatística descritiva e aplicado o teste qui-quadrado com nível de significância para $\mathrm{p}<0,05$. O projeto de pesquisa foi aprovado pelo Comitê de Ética em Pesquisa da Faculdade de Medicina de São José do Rio Preto com o protocolo n. 2712/2010.

\section{RESULTADOS}

Dos 500 usuários hipertensos caracterizados na análise de prontuários, 11 foram excluídos, pois durante as visitas domiciliares afirmaram não ser hipertensos e não realizarem nenhum tipo de tratamento. A maioria era do sexo feminino, faixa etária de 50 a 69 anos, com sobrepeso, de cor branca, aposentados e ensino fundamental incompleto (Tabela 1).

Observou-se que a maioria dos usuários realiza o tratamento anti-hipertensivo $(58,94 \%$ na Unidade A e 50,62\% na Unidade B). O abandono ao tratamento é de 33,74\%(83) na Unidade A e de 46,92\%(114) na Unidade B; o seguimento inadequado é de 7,32\%(18)e de 2,47\%(6), respectivamente.

Entre os 100 usuários hipertensos que abandonaram o tratamento ou realizavam inadequadamente o tratamento, selecionados aleatoriamente para realização das visitas domiciliares, a maioria era do gênero feminino (58\% e $62 \%$ nas Unidades A e B respectivamente), faixa etária entre 50 a 69 anos, sendo 68\% na unidade A e 56\% na B (Tabela 2) e com renda predominante de um a três salários mínimos.

Os dados relativos ao tempo de diagnóstico e tratamento estão apresentados nas tabelas 2 e 3. 
Observou-se que houve associação dos motivos de abandono ou seguimento inadequado do tratamento com a renda na Unidade $A(\mathrm{p}=0,002)$, pois os usuários de maior renda realizam tratamento em serviços de saúde privado, os demais em UBS. Houve também na Unidade A, significância da renda com a forma de aquisição $(p=0,007)$ e compra de medicamentos anti-hipertensivos $(p=034)$. Em relação a forma de aquisição, os usuários de menor renda adquirem os medicamentos pelo SUS e os de maior renda compram (Tabela 3).
Houve associação do tempo de hipertensão com número de medicamentos ingeridos; dos motivos do abandono ou seguimento inadequado do tratamento com número de medicamentos, percepção de PA elevada, forma de aquisição e compra de medicamentos anti-hipertensivos; do local em que realiza o tratamento com número de medicamentos, percepção de PA elevada, forma e aquisição de medicamentos; da quantidade de medicamentos com a forma de aquisição e compra dos mesmos. Mediante ao programa de análise estatística, não foi possível calcular o valor $\mathrm{p}$ de todas as variáveis (Tabela 4).

Tabela 1 - Distribuição das variáveis sócio demográficas dos usuários hipertensos cadastrados nas Unidades de Estratégia de Saúde da Família. Mirassol, 2010

\begin{tabular}{|c|c|c|c|c|}
\hline \multirow{2}{*}{ Variáveis } & \multicolumn{2}{|c|}{ Unidade A } & \multicolumn{2}{|c|}{ Unidade B } \\
\hline & $(n=246)$ & $(\%)$ & $(n=243)$ & $(\%)$ \\
\hline \multicolumn{5}{|l|}{ Sexo } \\
\hline Masculino & 96 & $39,02 \%$ & 93 & $38,27 \%$ \\
\hline Feminino & 150 & $60,98 \%$ & 150 & $61,73 \%$ \\
\hline \multicolumn{5}{|l|}{ Faixa Etária } \\
\hline 20 a 49 anos & 53 & $21,57 \%$ & 39 & $16,04 \%$ \\
\hline 50 a 69 anos & 143 & $58,10 \%$ & 131 & $54,00 \%$ \\
\hline Acima de 69 anos & 50 & $20,33 \%$ & 73 & $29,96 \%$ \\
\hline \multicolumn{5}{|l|}{ IMC } \\
\hline Abaixo do Peso & 4 & $1,80 \%$ & 0 & 0 \\
\hline IMC Normal & 55 & $22,52 \%$ & 41 & $17,11 \%$ \\
\hline Sobrepeso & 107 & $43,24 \%$ & 103 & $42,25 \%$ \\
\hline Obesidade & 80 & $32,44 \%$ & 99 & $40,64 \%$ \\
\hline \multicolumn{5}{|l|}{ Raça/cor } \\
\hline Branca & 186 & $75,57 \%$ & 192 & $79,09 \%$ \\
\hline Parda & 34 & $13,74 \%$ & 40 & $16,56 \%$ \\
\hline Negra & 26 & $10,69 \%$ & 11 & $4,35 \%$ \\
\hline \multicolumn{5}{|l|}{ Estado Civil } \\
\hline Casado(a) & 183 & $74,40 \%$ & 172 & $70,86 \%$ \\
\hline Viúvo(a) & 47 & $19,20 \%$ & 45 & $18,29 \%$ \\
\hline Divorciado(a) & 12 & $4,80 \%$ & 14 & $5,71 \%$ \\
\hline Solteiro(a) & 4 & $1,60 \%$ & 12 & $5,14 \%$ \\
\hline \multicolumn{5}{|l|}{ Ocupação } \\
\hline Aposentado(a) & 109 & $44,23 \%$ & 132 & $54 \%$ \\
\hline Do lar & 76 & $30,77 \%$ & 58 & $24,00 \%$ \\
\hline Outras & 61 & $25 \%$ & 53 & $22,00 \%$ \\
\hline \multicolumn{5}{|l|}{ Escolaridade } \\
\hline Analfabeto & 29 & $11,90 \%$ & 38 & $15,82 \%$ \\
\hline Alfabetizado & 9 & $3,71 \%$ & 8 & $3,39 \%$ \\
\hline Ens. F. Incompleto & 175 & $70,90 \%$ & 140 & $57,63 \%$ \\
\hline Ens. F. Completo & 19 & $7,94 \%$ & 27 & $11,30 \%$ \\
\hline Ens. Médio Incompleto & 4 & $1,59 \%$ & 10 & $3,95 \%$ \\
\hline Ens. Médio Completo & 8 & $3,17 \%$ & 14 & $5,65 \%$ \\
\hline Outros & 2 & $0,79 \%$ & 6 & $2,26 \%$ \\
\hline
\end{tabular}


Tabela 2 - Distribuição das variáveis do questionário realizado nas visitas domiciliares dos usuários hipertensos das Unidades de Saúde da Família. Mirassol, 2010

\begin{tabular}{|c|c|c|c|c|}
\hline \multirow[t]{2}{*}{ Variáveis } & \multicolumn{2}{|c|}{ Unidade A } & \multicolumn{2}{|c|}{ Unidade B } \\
\hline & $(n=246)$ & $(\%)$ & $(n=243)$ & $(\%)$ \\
\hline \multicolumn{5}{|l|}{ Tempo de Hipertensão } \\
\hline De 2 a 10 anos & 24 & $48 \%$ & 29 & $58 \%$ \\
\hline Mais de 10 anos & 26 & $52 \%$ & 21 & $42 \%$ \\
\hline \multicolumn{5}{|c|}{ Frequência de Aferição da PA } \\
\hline Diária ou Semanal & 9 & $18 \%$ & 7 & $14 \%$ \\
\hline Quinzenal & 13 & $26 \%$ & 7 & $14 \%$ \\
\hline Mensal & 5 & $10 \%$ & 5 & $10 \%$ \\
\hline Durante a consulta & 15 & $30 \%$ & 24 & $48 \%$ \\
\hline Quando sente-se mal & 8 & $16 \%$ & 7 & $14 \%$ \\
\hline \multicolumn{5}{|c|}{ Motivos do Abandono ou Seguimento inadequado } \\
\hline Tratamento serviço privado & 12 & $24 \%$ & 12 & $24 \%$ \\
\hline Tratamento em UBS & 29 & $58 \%$ & 30 & $60 \%$ \\
\hline Dificuldade de acesso & 5 & $10 \%$ & 2 & $4 \%$ \\
\hline Acha desnecessário & 4 & $8 \%$ & 6 & $12 \%$ \\
\hline \multicolumn{5}{|c|}{ Serviço de Saúde em que faz Tratamento } \\
\hline Privado & 13 & $26 \%$ & 12 & $24 \%$ \\
\hline Unidade Básica de Saúde & 29 & $58 \%$ & 30 & $60 \%$ \\
\hline Não faz tratamento & 8 & $16 \%$ & 8 & $16 \%$ \\
\hline
\end{tabular}

Tabela 3 - Distribuição das variáveis relativas ao uso de medicamentos entre usuários hipertensos das Unidades de Saúde da Família. Mirassol, 2010

\begin{tabular}{|c|c|c|c|c|}
\hline \multirow{2}{*}{ Variáveis } & \multicolumn{2}{|c|}{ Unidade A } & \multicolumn{2}{|c|}{ Unidade B } \\
\hline & $(n=50)$ & (\%) & $(n=50)$ & (\%) \\
\hline \multicolumn{5}{|c|}{ Quantidade de Medicamentos } \\
\hline Ingere um medicamento & 16 & $34,04 \%$ & 16 & $34,78 \%$ \\
\hline Ingere dois medicamentos & 19 & $40,43 \%$ & 15 & $32,61 \%$ \\
\hline Ingere mais de dois & 11 & $23,40 \%$ & 11 & $23,91 \%$ \\
\hline Somente quando passa mal & 1 & $2,13 \%$ & 4 & $8,70 \%$ \\
\hline Não ingere & 3 & & 4 & \\
\hline \multicolumn{5}{|c|}{ Forma de aquisição de Medicamentos } \\
\hline Adquire pelo SUS & 28 & $59,58 \%$ & 31 & $67,40 \%$ \\
\hline Necessita comprar & 17 & $36,17 \%$ & 13 & $28,26 \%$ \\
\hline Adquire de outra forma & 2 & $4,25 \%$ & 2 & $4,34 \%$ \\
\hline \multicolumn{5}{|l|}{ Compra de Medicamentos } \\
\hline Necessita comprar & 17 & $37,78 \%$ & 13 & $29,55 \%$ \\
\hline Não necessita comprar & 20 & $44,44 \%$ & 28 & $63,64 \%$ \\
\hline Compra algumas vezes & 8 & $17,78 \%$ & 3 & $6,81 \%$ \\
\hline \multicolumn{5}{|c|}{ Percepção do Aumento da PA } \\
\hline Percebe o aumento da PA & 33 & $66 \%$ & 31 & $62,00 \%$ \\
\hline Não Percebe & 17 & $34 \%$ & 19 & $38,00 \%$ \\
\hline \multicolumn{5}{|c|}{ Atitude tomada quando percebe Aumento da PA } \\
\hline Procura atendimento & 14 & $42,42 \%$ & 8 & $25,81 \%$ \\
\hline Ingere medicamentos & 10 & $30,30 \%$ & 9 & $29,03 \%$ \\
\hline Outros & 9 & $27,28 \%$ & 14 & $45,16 \%$ \\
\hline
\end{tabular}


Tabela 4 - Valores p do teste associativo para as variáveis avaliadas durante as visitas domiciliares dos usuários hipertensos das Unidades de Saúde da Família. Mirassol, 2010

\begin{tabular}{|c|c|c|c|c|c|c|c|c|c|c|}
\hline \multirow[t]{2}{*}{$\begin{array}{c}\text { Variáveis } \\
\text { resposta }\end{array}$} & \multicolumn{2}{|c|}{$\begin{array}{l}\text { Número de } \\
\text { medicamentos }\end{array}$} & \multicolumn{2}{|c|}{$\begin{array}{c}\text { Percepção da PA } \\
\text { elevada }\end{array}$} & \multicolumn{2}{|c|}{ Atitude tomada } & \multicolumn{2}{|c|}{$\begin{array}{l}\text { Aquisição de } \\
\text { medicamentos }\end{array}$} & \multicolumn{2}{|c|}{$\begin{array}{c}\text { Compra de } \\
\text { medicamentos }\end{array}$} \\
\hline & $\mathbf{A}$ & B & $\mathbf{A}$ & B & $\mathbf{A}$ & B & $\mathbf{A}$ & B & $\mathbf{A}$ & B \\
\hline $\begin{array}{l}\text { Tempo de } \\
\text { hipertensão }\end{array}$ & 0,877 & 0,005 & 0,924 & 0,562 & 0,342 & 0,206 & 0,372 & 0,2 & 0,769 & 0,05 \\
\hline $\begin{array}{l}\text { Frequência } \\
\text { de aferição } \\
\text { da PA }\end{array}$ & 0,444 & 0,093 & 0,811 & 0,75 & - & 0,517 & 0,806 & 0,289 & - & 0,205 \\
\hline $\begin{array}{l}\text { Motivos do } \\
\text { abandono }\end{array}$ & & & & & & & & & & \\
\hline $\begin{array}{l}\text { ou segui- } \\
\text { mento ina- } \\
\text { dequado }\end{array}$ & 0,002 & 0,001 & 0,195 & 0,047 & 0,417 & - & $<0,001$ & - & $<0,001$ & $<0,001$ \\
\hline $\begin{array}{l}\text { Instituição } \\
\text { que faz tra- } \\
\text { tamento }\end{array}$ & $<0,001$ & - & 0,017 & 0,235 & 0,235 & - & $<0,001$ & - & $<0,001$ & $<0,001$ \\
\hline $\begin{array}{l}\text { Quantidade } \\
\text { de medica- } \\
\text { mentos }\end{array}$ & - & - & 0,132 & 0,65 & 0,967 & 0,447 & 0,003 & $<0,001$ & 0,012 & 0,001 \\
\hline $\begin{array}{l}\text { Percepção } \\
\text { da PA ele- } \\
\text { vada }\end{array}$ & - & - & - & - & - & - & 0,182 & 0,852 & 0,517 & 0,966 \\
\hline $\begin{array}{l}\text { Atitude to- } \\
\text { mada }\end{array}$ & - & - & - & - & - & - & 0,682 & 0,917 & 0,764 & 0,537 \\
\hline $\begin{array}{l}\text { Aquisição } \\
\text { dos medi- } \\
\text { camentos }\end{array}$ & - & - & - & - & - & - & - & - & - & - \\
\hline
\end{tabular}

\section{DISCUSSÃO}

Neste estudo, a prevalência de hipertensos, em ambas as unidades foi do gênero feminino em relação ao masculino. As mulheres procuram mais os serviços de saúde do que os homens, uma vez que são donas de casas ou aposentadas, estando disponíveis aos horários de atendimento das unidades de saúde ${ }^{(8-9)}$.

O perfil epidemiológico de uma população é alterado mediante ao envelhecimento dessa população e dentre as de destaque temos as doenças crônicas não-transmissíveis (DCNT), que causam milhões de mortes a cada ano em todo o mundo ${ }^{(10)}$.

A população mais acometida pela HAS é a idosa, como foi observado neste estudo, corroborando com uma pesquisa também realizada em Unidade de Saúde da Família que observou a prevalência de não adesão dos idosos ao pro- grama de hipertensão de $8,5 \%^{(11)}$.

Em um estudo sobre perfil de hipertensos em Unidade de Saúde da Família constatou-se prevalência de usuários com IMC de sobrepeso, ou seja, mais de $70 \%$ dos indivíduos hipertensos estavam acima do peso, estando de acordo com os resultados desta pesquisa ${ }^{(12)}$. No que se refere ao tempo de hipertensão, houve congruência com resultados de um estudo sobre conhecimento sobre o estilo de vida saudável no controle de pessoas hipertensas, em que o tempo de HAS foi maior que $5 \operatorname{anos}^{(13)}$.

Em relação à frequência de aferição, foi predominante no momento da consulta ao cardiologista, seja em instituição privada ou em UBS, não congruente com estudo sobre adesão ao tratamento anti-hipertensivo e fatores associados na área de abrangência de Unidade de Saúde da Família, realizado em Londrina-Paraná, em que $77,4 \%$ dos hipertensos relataram ter sua pressão aferida pelo 
menos uma vez ao mês ${ }^{(14)}$.Verificou-se que a maioria dos usuários segue o tratamento anti-hipertensivo. Entretanto, um estudo sobre fatores associados à não-adesão do tratamento com anti-hipertensivos em pessoas atendidas em unidades de saúde da família, observou valor superior de não adesão $(53,1 \%)^{(15)}$.

Os dados deste estudo são semelhantes ao da pesquisa realizada sobre os motivos relacionados à não adesão onde se constatou os motivos como a opção por plano de saúde privado, a dificuldade de se deslocar até o serviço de saúde, interrupção do tratamento por se sentirem bem e por falta de tempo ${ }^{(16)}$.

Em relação à renda dos usuários hipertensos evidenciou-se que a predominância foi de um a três salários mínimos e os motivos relacionados ao abandono ou seguimento inadequado apresentou associação à renda, na unidade $\mathrm{A}$, pressupondo que os de maior renda realizam acompanhamento em instituição privada, enquanto os de menor renda, nas UBS.

O baixo custo dos medicamentos está envolvido na adesão, além de outros aspectos como não conseguir acompanhar um tratamento ao longo do tempo, em virtude da dificuldade de acesso ao sistema público e pela situação financeira da maioria dos doentes impossibilitando a procura de assistência em serviços privados, sendo que a renda nesse caso está interferindo ${ }^{(15)}$, entretanto, a situação financeira no presente estudo não está ligada ao abandono ou seguimento inadequado do tratamento definitivo e sim à opção de tratamento em instituição privada ou UBS.

Em estudo de revisão relacionado ao regime terapêutico, evidenciou-se que vários fatores interferem na adesão ao tratamento, como: complexidade do regime terapêutico, dosagem, horário, duração do tratamento, entre outros ${ }^{(17)}$. Esses resultados não coincidiram com os achados deste estudo, onde o motivo estava relacionado à instituição de acompanhamento e não à dificuldade em aderir à terapêutica.

Houve significância entre os motivos de abandono ou seguimento inadequado com a percepção de aumento da pressão arterial na unidade B, uma vez que os usuários que realizavam tratamento anti-hipertensivo com cardiologista em instituição particular ou em UBS percebiam mais a elevação da pressão arterial em relação aos que não realiza- vam o tratamento em nenhuma instituição, como aqueles que relataram achar dificuldade ao acesso ou acharem desnecessário o tratamento.

Ainda em relação aos motivos de abandono ou seguimento inadequado do tratamento antihipertensivo, houve associação entre os motivos e forma de aquisição de medicamentos em ambas as Unidades de Saúde da Família com necessidade de compra de medicamentos. Na unidade A encontrou os hipertensos que tratavam com cardiologistas em instituições particulares e compravam todos os seus medicamentos e na unidade $\mathrm{B}$ a maioria adquire pelo SUS os seus medicamentos.

Em estudo realizado sobre o local de aquisição desses medicamentos, $54,7 \%$ foram obtidos em unidades básicas de saúde, ou seja, pelo $\operatorname{SUS}^{(18)}$, semelhantes aos dados do estudo, em que $59,58 \%$ dos usuários da unidade A adquirem pelo SUS e $67,40 \%$ da B, havendo menor percentual dos usuários que tratam em UBS e que necessitam comprar seus medicamentos.

É importante destacar o fato de que o abandono ou seguimento inadequado do tratamento não necessariamente foi não adesão ao tratamento, uma vez que a maioria dos usuários, ou seja, mais de $80 \%$ deles estava realizando tratamento em outra instituição, seja pública ou privada. Pesquisa sobre motivos do abandono do seguimento médico no cuidado a portadores de hipertensão arterial corroborou com estes achados, devido $56 \%$ dos entrevistados estarem frequentando outros serviços de saúde para o tratamento da hipertensão ${ }^{(19)}$.

Os usuários hipertensos por muitas vezes não apresentarem sintomas, acabam abandonando o tratamento, retomando-o apenas após a crise hipertensiva, porém, outros hipertensos realizam o tratamento regularmente na prevenção de complicações ${ }^{(20)}$. Neste estudo, a maioria dos hipertensos era sintomático, tanto os que não tratavam quanto os que eram acompanhados em instituição privada ou em UBS.

\section{CONSIDERAÇÕES FINAIS}

Os usuários hipertensos que abandonaram ou não seguem adequadamente o tratamento anti-hipertensivo possuem características sociodemográficas semelhantes aos descritos na literatura.

Os principais motivos relacionados ao abandono ou seguimento inadequado do tratamento 
anti-hipertensivo foram: realização do tratamento com cardiologista, dificuldade de acesso à Unidade de Saúde da Família e por não achar necessário o tratamento. Portanto, a maioria dos usuários não abandonou o seu tratamento definitivamente e sim optaram em realizar o tratamento com médico cardiologista, profissional que não se encontra na equipe da Unidade de Saúde da Família do município referido.

\section{REFERÊNCIAS}

1. Carlos PR, Palha PF, Veiga EV, Beccaria LM. Perfil de hipertensos de um núcleo de saúde da família. Arq. ciênc. saúde. 2008;15(4):176-81.

2. Faria IBR, Ventura JC, Silva DE, Moretti-Pires RO. O processo de trabalho em saúde da família no contexto do interior da Amazônia. Cogitare enferm. 2010;15(2):231-7.

3. Costa JMBS, Silva MRF, Carvalho EF. Avaliação da implantação da atenção à hipertensão arterial pelas equipes de Saúde da Família do município do Recife (PE, Brasil). Ciên. saude colet. [Internet]. 2011 [acesso em 16 jan 2012];16(2). Disponível: http://www.scielosp.org/pdf/csc/v16n2/v16n2a26. pdf

4. Zaitune MPA, Barros MBA, César CLG, Carandina L, Goldbaum M. Hipertensão arterial em idosos: prevalência, fatores associados e práticas de controle no Município de Campinas, São Paulo, Brasil. Cad. saude publica. 2006;22(2):285-94.

5. Calista AA, Vaconcelos ASS, Oliveira MRL. Hipertensão arterial sistêmica: fatores contribuintes para a adesão ao tratamento. Rev. tema. 2008;7(1011):101-10.

6. Jardim PCBV, Jardim T de SV. Modelos de estudos de adesão ao tratamento anti-hipertensivo. Rev. bras. hipertens. 2006;13(1):26-9.

7. Instituto Brasileiro de Geografia e Estatística [home page Internet]. 2011 Ago. [acesso em 01 ago 2011]. São Paulo. Mirassol, SP; Disponível : http://www. ibge.gov.br/cidadesat/topwindow.htm?1

8. Veras RFS, Oliveira JD. Aspectos sócio-demográficos que influenciam na adesão ao tratamento antihipertensivo. Rev. RENE. 2009;10(3):132-8

9. Tacon KCB, Santos HCO, Castro EC. Perfil epidemiológico da hipertensão arterial sistêmica em pacientes atendidos em hospital público. Rev. soc. bras. clín. méd. 2010;8(6):486-9.

10. Felipe GF, Abreu RNDC, Moreira TMM. Aspectos contemplados na consulta de enfermagem ao paciente com hipertensão atendido no Programa Saúde da Família. Rev. esc. enferm. USP. 2008;42(4):620-7.

11. Contiero AP, Pozati MPS, Challouts RI, Carreira L, Marcon SS. Idoso com hipertensão arterial: dificuldades de acompanhamento na Estratégia Saúde da Família. Rev. gaúcha enferm. 2009;30(1):6270 .

12. Oliveira EA, Bubach S, Flegeler DS. Perfil de hipertensos em uma Unidade de Saúde da Família. Rev. enferm.UERJ. 2009;17(3):383-7.

13. Serafim T, Jesus ES, Pierin AMG. Influência do conhecimento sobre o estilo de vida saudável no controle de pessoas hipertensas. Acta paul. enferm. 2010;23(5):658-64.

14. Girotto E. Adesão ao tratamento anti-hipertensivo e fatores associados na área de abrangência de uma Unidade de Saúde da Família [dissertação]. Londrina: Universidade Estadual de Londrina; Jun; 2008.

15. Santa-Helena ET, Nemes MIB, Neto JE. Fatores associados à não-adesão ao tratamento com anti-hipertensivos em pessoas atendidas em unidades de saúde da família. Cad. saude publica. 2010;26(12):2389-98.

16. Oshiro ML, Castro LLC, Cymrot R. Fatores para não-adesão ao programa de controle da hipertensão arterial em Campo Grande, MS. Rev. ciênc. farm. básica apl. 2010;31(1):95-100.

17. Gusmão JL, Ginani GF, Silva GV, Ortega KC, Jr. Mion D. Adesão ao tratamento em hipertensão arterial sistólica isolada. Rev. bras. hipertens. 2009;16(1):38-43.

18. Vosgerau MZS, Cabrera MAS, Souza RKT. Saúde da Família e utilização de medicamentos antihipertensivos e antidiabéticos. Rev. bras. cardiol. 2011;24(2):95-104.

19. Duarte MTC, Cyrino AP, Cerqueira ATAR, Nemes MIB, Iyda M. Motivos do abandono do seguimento médico no cuidado a portadores de hipertensão arterial: a perspectiva do sujeito. Ciênc. saude 
coletiva. 2010;15(5):2603-10.

20. Manfroi A, Oliveira FA. Dificuldades de adesão ao tratamento na hipertensão arterial sistêmica: considerações a partir de um estudo qualitativo em uma unidade de Atenção Primária à Saúde. Rev. bras. med. fam. comunidade. 2006;2(7):165-76. 